Research Paper

\title{
Exercise Preconditioning Attenuates Neurological Injury by Preserving Old and Newly Formed HSP72- Containing Neurons in Focal Brain Ischemia Rats
}

\author{
Yu-Lin Wang1,2,3, Cheng-Hsien Lin ${ }^{4}$, Chi-Chun Chen 5 , Ching-Ping Chang, ${ }^{6}$ Kao-Chang Lin 7 , Fong-Chin
} $\mathrm{Su}^{1,8}{ }^{\bowtie}$, Willy Chou ${ }^{3,9}{ }^{\bowtie}$

1. Department of Biomedical Engineering, National Cheng Kung University, Tainan, Taiwan

2. Center of General Education, Southern Taiwan University of Science and Technology, Tainan, Taiwan

Department of Physical Medicine and Rehabilitation, Chi-Mei Medical Center, Tainan, Taiwan

Department of Medicine, Mackay Medical College, New Taipei, Taiwan

Department of Electronic Engineering, National Chin-Yi University of Technology, Taichung, Taiwan

Department of Medical Research, Chi Mei Medical Center, Tainan, Taiwan

Department of Neurology, Chi Mei Medical Center, Tainan, Taiwan

Medical Device Innovation Center, National Cheng Kung University, Tainan, Taiwan

Department of Recreation and Healthcare Management, Chia Nan University of Pharmacy and Science, Tainan, Taiwan

$\triangle$ Corresponding authors: Fong-Chin Su, PhD, Department of Biomedical Engineering, National Cheng Kung University, Tainan, Taiwan Mailing address: No.1, University Road, Tainan City 701, Taiwan Phone: +886-6-2757575-63400 Fax: +886-6-2343270 E-mail: fcsu@mail.ncku.edu.tw Willy Chou, MD, Department of Physical Medicine and Rehabilitation, Chi-Mei Medical Center, Tainan, Taiwan Mailing address: No. 901, Zhonghua Rd., Yongkang District, Tainan City 710, Taiwan Phone: +886-6-2812811 Fax: +886-6-2832639 E-mail: ufan0101@ms22.hinet.net

(C) Ivyspring International Publisher. This is an open access article distributed under the terms of the Creative Commons Attribution (CC BY-NC) license (https://creativecommons.org/licenses/by-nc/4.0/). See http://ivyspring.com/terms for full terms and conditions.

Received: 2019.01.09; Accepted: 2019.04.04; Published: 2019.05.10

\begin{abstract}
Background: Exercise preconditioning $\left(\mathrm{EP}^{+}\right)$is a useful and important procedure for the prevention of stroke. We aimed to ascertain whether $\mathrm{EP}^{+}$protects against ischemic brain injury by preserving heat shock protein (HSP) 72-containing neurons in ischemic brain tissues.

Methods: Adult male Sprague-Dawley rats $(n=240)$ were used to assess the contribution of HSP72-containing neurons to the neuroprotective effects of $\mathrm{EP}^{+}$on ischemic brain injury caused by transient middle cerebral artery occlusion.

Results: Significant $(\mathrm{P}<0.05)$ increases in the percentages of both old HSP72-containing neurons (NeuN+HSP72 double positive cells) (18 20\% vs. 40 50\%) and newly formed HSP72-containing neurons (BrdU+NeuN+HSP72 triple positive cells); (2 3\% vs. 16 20\%) after 3 weeks of exercise coincided with significant $(\mathrm{P}<0.05)$ reductions in brain ischemia volume $\left(250 \mathrm{~mm}^{3}\right.$ vs. $\left.100 \mathrm{~mm}^{3}\right)$, brain edema $(78 \%$ vs. $74 \%$ brain water content), blood-brain barrier disruption $(1.5 \mu \mathrm{g} / \mathrm{g}$ vs. $0.7 \mu \mathrm{g} / \mathrm{g}$ tissue Evans Blue dye extravasation) and neurological motor deficits (neurological severity scores of 12 vs. 6 and maximal angles of $60^{\circ}$ vs. $20^{\circ}$ ) in brain ischemia rats. Reductions in the percentages of both old (from 40 50\% to $10 \sim 12 \%$ ) and newly formed (from 18 20\% to 5 7\%) HSP72-containing neurons by gene silencing with an intracerebral injection of PSUPER small interfering RNA showed a significant $(P<0.05)$ reversal in the neuroprotective outcomes. Our data provide an inverse correlation between the $\mathrm{EP}^{+}$-mediated increases in both old and newly formed HSP72-containing neurons and the extent of cerebral ischemic injury.

Conclusions: The percentages of both old and newly formed HSP72-containing neurons are inversely correlated with the outcomes of ischemic brain injury. Additionally, preischemic treadmill exercise improves the outcomes of ischemic brain injury by preserving both the old and newly formed HSP72-containing neurons in rats.
\end{abstract}

Key words: Exercise, heat shock protein 72, neuroprotection, ischemic brain injury

\section{Introduction}

The American Heart Association/American Stroke Association has promoted that the primary

prevention of stroke is particularly important [1]. Exercise preconditioning $\left(\mathrm{EP}^{+}\right)$is widely believed to 
be a safe and effective preventive measure for people who undergo a stroke [2]. Clinical data have also shown that physical inactivity before stroke predicts a higher risk of being dependent both before and after stroke $[3,4]$. Although preischemic $\mathrm{EP}^{+}$can attenuate neurobehavioral deficits after ischemic brain injury [5], the exact neuroprotective mechanisms of preischemic $\mathrm{EP}^{+}$against cerebral ischemic injury remain unclear.

In the normal rat brain, there is little to no expression of inducible heat shock protein (HSP) 72 [6]. However, following occlusion of the middle cerebral artery, HSP72 induction in the ischemic core, or penumbra, occurs primarily in neurons, but HSP72 can also be expressed in astrocytes, microglia and endothelial cells $[6,7]$. On the other hand, $\mathrm{EP}^{+}$ increases neuronal and astroglial levels of HSP72 in the gray matter of spinal cord tissue in normal rats [8]. Transfection and viral overexpression of HSP72 in neurons and glia protects against in vitro ischemia [6] and in vivo ischemia [8,9]. Additionally, knocking out HSP72 worsens the outcomes and transgenic overexpression improves the outcomes of cerebral ischemia [10]. Pharmacological induction of HSP72 protects against cerebral ischemia [11]. Although it is well known that $\mathrm{EP}^{+}$reduces neuronal apoptosis in stroke rats by upregulating HSP72 in the brain [12], it is unknown whether $\mathrm{EP}^{+}$increases neuronal expression of HSP72 in normal rat brain tissues and protects against ischemic brain injury in rats by preserving old and newly formed HSP72-containing neurons.

In the present study, we first used immunofluorescence staining methods to elucidate whether old and/or newly formed HSP72-containing neurons can be upregulated by preischemic $\mathrm{EP}^{+}$in rats with or without middle cerebral artery occlusion (MCAO). Second, we describe the contributions of old and/or newly formed HSP72-containing neurons to $\mathrm{EP}^{+}$-mediated attenuation of neurological injury (including brain infarct, neurological motor deficits, blood-brain barrier (BBB) disruption, and brain edema) in a ischemic brain injury model in rats [13]. To inhibit HSP72 expression in the brain tissue, the ischemic brains were intraoperatively microinjected with pSUPER plasmid expressing HSP72 small interfering RNA (siRNA-HSP72) [12, 14].

\section{Materials and methods}

\section{Animals and stroke model}

Two hundred forty adult male Sprague-Dawley rats (weight, $248 \pm 15 \mathrm{~g}$ ) were housed under controlled environmental conditions with an ambient temperature of $24 \pm 2^{\circ} \mathrm{C}$, a relative humidity of $65 \%$ and a 12-h light/dark cycle, with free access to food and water. Three weeks after treadmill $\mathrm{EP}^{+}$, ischemic brain injury was induced by MCAO in rats by intraluminal filaments, using the relatively noninvasive technique detailed previously [15]. The protocol was approved by the Institutional Review Board for Animal Care and Use (Assurance Number: 100120755).

Recombinant pSUPER plasmid expressing siRNA-HSP72 constructed using the pSUPER vector (Oligo Engine, Seattle, WA, USA), which contains the polymerase-III H1-RNA gene promoter and can direct the synthesis of siRNA-like transcripts. The target sequence for HSP72 (Gen Bank Accession No. NM-0319712) was chemically synthesized (Tri-1 Biotech, Taipei, Taiwan) as complementary oligonucleotides. The synthetic oligonucleotides of siRNA-HSP72 5'-GATCCCCGGAGATCATCGCCAA CGACTAAGAGAGTTGGCGATGATCTCCTTTTTG GAAA-3' and 3'-GGGCCTCTAGTAGCGGTTGCTG AAGTTCTCTCAGCAACCGCTACTAGAGGAAAA ACCTTTTCGAA-5' were annealed and cloned downstream of the $\mathrm{H} 1$ promoter to construct a recombinant pSUPER HSP72 plasmid. The cloned HSP72 target sequence was sequence-confirmed using a DNA sequencer (ABI Prism 377, Applied Biosystems, Foster City, CA, USA). During the MCAO surgery, an acute dose of siRNA-HSP72 $(12.5 \mu \mathrm{g} / \mathrm{rat}$ in $25 \mu \mathrm{l}$ of pSUPER RNAi delivery media [siRNA-vector]) was microinjected into the frontal cortex at a flow rate of $0.5 \mu \mathrm{l} / \mathrm{min}$ using a microinfusion pump (CMA 100, Carnegie Medicine $\mathrm{AB}$, Stockholm, Sweden) according to the coordinates of the atlas of Paxinos and Watson [16]. A single 28-gauge stainless steel injection cannula was lowered into the right frontal cortex (coordinates: $12 \mathrm{~mm}$ anterior to bregma, $4.6 \mathrm{~mm}$ lateral to midline and 3.0 $\mathrm{mm}$ ventral to the skull surface).

\section{Exercise preconditioning $\left(\mathrm{EP}^{+}\right)$protocol}

Animals were trained on a treadmill (model Exer-3/6, Columbus Instruments, Columbus, $\mathrm{OH}$, USA) 5 days a week for 3 weeks. They were acclimatized to run for $15 \mathrm{~min}$ at $20 \mathrm{~m} / \mathrm{min}$ at $0^{\circ}$ for 3 days initially, and then they were running for $30 \mathrm{~min}$ at $20 \mathrm{~m} / \mathrm{min}, 30 \mathrm{~min}$ at $30 \mathrm{~m} / \mathrm{min}$ and $60 \mathrm{~min}$ at 30 $\mathrm{m} / \mathrm{min}$ after 1,2 and 3 weeks of training, respectively. Nonexercise preconditioning controls (EP-) were placed daily on a stationary treadmill and were given electrical stimulation in a manner identical to that used for the $\mathrm{EP}^{+}$group.

\section{Exercise groups and procedures}

The animals were randomly assigned to one of eight groups: (i) $\mathrm{EP}^{-}+\mathrm{MCAO}^{-}$rats that received an 
intracerebral injection of siRNA-vector (EP-+ $\mathrm{MCAO}+$-siRNA-vector), (ii) EP-+MCAO-+siRNAHSP72, (iii) $\mathrm{EP}^{+}+\mathrm{MCAO}^{-+}$siRNA-vector, (iv) $\mathrm{EP}^{++}$ $\mathrm{MCAO}^{-}+$siRNA-HSP72, (v) $\mathrm{EP}^{-}+\mathrm{MCAO}^{+}+\mathrm{siRNA}-$ vector, (vi) $\mathrm{EP}^{-}+\mathrm{MCAO}^{+}+$siRNA-HSP72, (vii) $\mathrm{EP}^{++}$ $\mathrm{MCAO}^{+}+$siRNA-vector, and (viii) $\mathrm{EP}^{+}+\mathrm{MCAO}^{+}+$ siRNA-HSP72. The experimental procedures in this study are shown in Figure 1. Of all the rats in this study, 13 rats were excluded because of death prior to sacrifice, including 0 in the EP-+MCAO-+ siRNA-vector group (i); 0 in the $\mathrm{EP}^{-}+\mathrm{MCAO}^{-+}$ siRNA-HSP72 group (ii); 0 in the $\mathrm{EP}^{++} \mathrm{MCAO}^{-+}$ siRNA-vector group (iii); 0 in the $\mathrm{EP}^{++} \mathrm{MCAO}^{-+}$ siRNA-HSP72 group (iv); 4 in the $\mathrm{EP}-\mathrm{MCAO}^{+}+$ siRNA-vector group (v); 4 in the $\mathrm{EP}-\mathrm{MCAO}^{+}+$ siRNA-HSP72 group (vi); 2 in the $\mathrm{EP}^{+}+\mathrm{MCAO}^{+}+$ siRNA-vector group (vii); 3 in the $\mathrm{EP}^{+}+\mathrm{MCAO}^{++}$ siRNA-HSP72 group (viii).

In experiment 1 , the percentages of cortical and striatal old HSP72-containing neurons (i.e., HSP72+NeuN+DAPI-positive cells) were determined 3 days after the MCAO or sham operation in all groups (Figure 2).

In experiment 2, the percentages of newly formed neurons containing HSP72 (i.e., HSP72+NeuN+BrdU+DAPI-positive cells) in cortical and striatal tissue of the ipsilateral brain were determined 3 days after MCAO or sham operation in all groups (Figure 3). We examined the same regions of the right frontal cortex and the striatum in both the ischemic brains and nonischemic brains.

In experiment 3 , the neurobehavioral functions were determined 1 day before the injury and 3 days after injury in all groups (Figure 4).

In experiment 4 , the cerebral infarct volumes were determined 3 days after the MCAO or sham operation in all groups (Figure 5).

In experiment 5, the BBB permeability and Evans Blue dye extravasation were determined 3 days after the MCAO or sham operation in all groups (Figure 6).

\section{Neurobehavioral function test}

Acute neurological motor deficits were assessed in all groups the day before and 3 days after MCAO or sham injury using both a neurological severity score test [17] and an inclined system with a microcontroller [18]. The former reveals a composite of the motor, sensory, and reflex test scores, whereas the latter measures limb strength. The modified Neurological Severity Score (mNSS), which comprises tests of motor, sensory, beam balance, reflex absence and abnormal movement functions, was used to evaluate the neurological function of the rats before and after the operation. Neurological function was graded on a scale of $0-18$ : 1-6, mild injury; 7-12, moderate injury;
13-18, severe injury. The inclined plane was used to measure limb strength. The inclined plane used in this study was constructed of a $60 \mathrm{~cm} \times 60 \mathrm{~cm}$ adjustable acrylic panel. A motor and a ball screw were used to control the inclined angle of the acrylic plane from $0^{\circ}$ (horizontal) to $70^{\circ}$ [18]. The angle was increased or decreased in $5^{\circ}$ increments to determine the maximal angle at which an animal could maintain balance on the plane. The data for each day were the mean values of the left- and right-side limb maximal angles.

\section{Assessment of cerebral infarct}

The rats were deeply anesthetized, and brains were rapidly removed, frozen in liquid nitrogen and then sectioned for immunohistochemistry. Brain sections were stained with 2,3,5-triphenyl-tetrazolium chloride (TTC) as detailed previously [19]. The infarct volume $\left(\mathrm{mm}^{3}\right)$ was measured in each slice and summed using computerized planimetry [20]. We also adopted the method of Lin et al. [21] to correct the distortion of infarct volume caused by brain edema.

\section{Bromodeoxyuridine labeling}

Bromodeoxyuridine (BrdU), a thymidine analog that is incorporated into the DNA of dividing cells during the S-phase, was used for mitotic labeling (Roche Diagnostics, Indianapolis, IN, USA; $50 \mathrm{mg} / \mathrm{kg}$ ) as described previously [22]. BrdU was administered intraperitoneally immediately at the beginning of surgery and once daily for 3 consecutive days. Three days after MCAO, brains were removed for BrdU labeling. The BrdU immunostaining procedure with a specific antibody against BrdU (1:400; Roche Diagnostics) and the quantification of BrdU-immunoreactive cells were described previously [22].

\section{Immunofluorescence staining}

Three days after MCAO, rat brains were prepared as described above. The brains were embedded in paraffin and serially sectioned $(10 \mu \mathrm{m})$ from the frontal cortex to the commencement of the hippocampus by a research assistant who was blinded to the study protocol. Every 10th section was then collected for immunofluorescence staining. For each animal, 6 serial coronal sections with $100-\mu$ m intervals were collected from the cortex. Sections were incubated in $2 \mathrm{~mol} / \mathrm{L} \mathrm{HCl}$ for $30 \mathrm{~min}$, rinsed in 0.1 mol/L boric acid ( $\mathrm{pH}$ 8.5) for $3 \mathrm{~min}$ at room temperature, and then incubated with primary antibodies in phosphate-buffered saline (PBS) containing $0.5 \%$ normal bovine serum at $4^{\circ} \mathrm{C}$ overnight; secondary antibodies were incubated for 1 $\mathrm{h}$ at room temperature. The sections were cover slipped with mounting medium (Fluorescent Mounting Medium; Dako). The labeled cells were 
calculated in 5 coronal sections from each rat and are expressed as the mean number of cells per section. For negative control sections, all the procedures were performed without the primary antibody. The primary and secondary antibodies for multiple staining are listed in Table 1.

\section{Brain water content and Evans blue dye extravasation assays}

The water content of the ipsilateral brains was evaluated via the wet weight/dry weight ratio method, as previously reported [23]. Evans blue dye extravasation assays were performed in accordance with the methods of Lin et al. [24]. Three hours before sacrifice, animals received an intravenous dose of Evans Blue dye $(4 \%, 0.25 \mathrm{ml} / \mathrm{rat})$. The quantity of extravasated Evans Blue dye was detected by a spectrophotometer at $610 \mathrm{~nm}$ and quantified according to a standard curve. The results are presented as $\mu \mathrm{g}$ of Evans Blue dye per $\mathrm{g}$ of tissue.

\section{Statistical analysis}

GraphPad Prism (version 7.01 for Windows; GraphPad Software, San Diego, CA, USA) was used for all statistical analyses. Data are expressed as the means \pm standard deviation (SD). Statistical analysis was performed using two-way analysis of variance (ANOVA) with Tukey's and Bonferroni post hoc testing to analyze the percentage of infarct area and the behavioral performance, respectively. Histological measures were analyzed using one-way ANOVA with Bonferroni post hoc tests. A value of $\mathrm{P}<0.05$ was considered statistically significant.

Table 1. Immunofluorescent staining in a focal ischemic injury rat model.

\begin{tabular}{|c|c|c|c|c|c|}
\hline Antibody & Antigen & Host & Company & Catalog\# & Dilution \\
\hline \multicolumn{6}{|l|}{ Primary Antibody } \\
\hline NeuN & Neuron & Mouse & Millipore & MAB377 & $1: 400$ \\
\hline HSP72 & HSP72 & Rabbit & Abgent & ASM10001 & $1: 200$ \\
\hline DAPI & Nucleic acid & & Invitrogen & D1306 & $1: 20000$ \\
\hline BrdU & Bromodeoxyuridine & Rat & Abcam & Ab6326 & $1: 200$ \\
\hline \multicolumn{6}{|l|}{ Secondary Antibody (conjugation) } \\
\hline Mouse IgG (Alexa Fluor 568) & Mouse IgG & Goat & Invitrogen & A11031 & $1: 200$ \\
\hline Rabbit IgG (Alexa Fluor 488) & Rabbit IgG & Goat & Invitrogen & A11034 & $1: 200$ \\
\hline
\end{tabular}

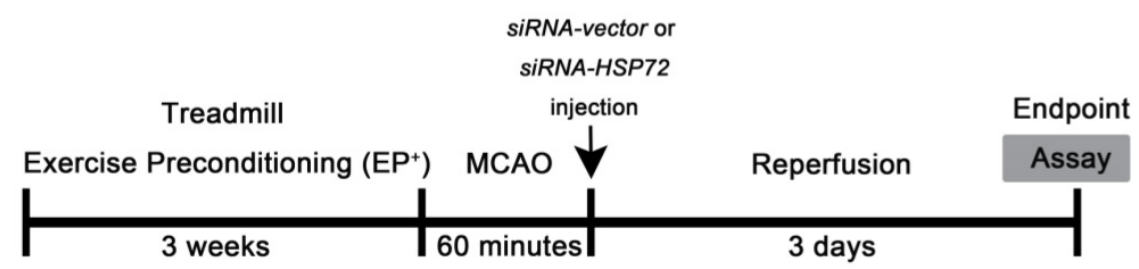

240 rats

\begin{tabular}{|c|c|c|c|c|c|c|c|c|}
\hline & EP'+ & $E P^{-+}$ & $\mathrm{EP}^{++}$ & $\mathrm{EP}^{++}$ & EP- & EP- + & $\mathrm{EP}^{++}$ & $\mathrm{EP}^{\prime+}+$ \\
\hline & $\begin{array}{c}\text { MCAO+ } \\
\text { siRNA-vector }\end{array}$ & $\begin{array}{c}\text { MCAO+ } \\
\text { siRNA-HSP72 }\end{array}$ & $\begin{array}{l}\text { MCAO+ } \\
\text { siRNA-vector }\end{array}$ & $\begin{array}{c}\text { MCAO+ } \\
\text { siRNA-HSP72 }\end{array}$ & $\begin{array}{l}\text { MCAO'+ } \\
\text { siRNA-vector }\end{array}$ & $\begin{array}{c}\text { MCAO'+ } \\
\text { siRNA-HSP72 }\end{array}$ & $\begin{array}{l}\text { MCAO'+ } \\
\text { siRNA-vector }\end{array}$ & $\begin{array}{c}\text { MCAO++ } \\
\text { siRNA-HSP72 }\end{array}$ \\
\hline & 30 rats & 30 rats & 30 rats & 30 rats & $\begin{array}{c}26 \text { rats } \\
\text { (4/30 died) }\end{array}$ & $\begin{array}{c}26 \text { rats } \\
\text { (4/30 died) }\end{array}$ & $\begin{array}{c}28 \text { rats } \\
\text { (2/30 died) }\end{array}$ & $\begin{array}{c}27 \text { rats } \\
\text { (3/30 died) }\end{array}$ \\
\hline $\begin{array}{c}\text { TTC staining } \\
\text { and neurological } \\
\text { evaluation }\end{array}$ & $n=10$ & $n=10$ & $n=10$ & $n=10$ & $n=8$ & $n=8$ & $n=9$ & $n=9$ \\
\hline $\begin{array}{c}\text { Brain water } \\
\text { content }\end{array}$ & $n=6$ & $n=6$ & $n=6$ & $n=6$ & $n=6$ & $n=6$ & $n=6$ & $n=6$ \\
\hline $\begin{array}{l}\text { Evans Blue } \\
\text { extravasation }\end{array}$ & $n=6$ & $n=6$ & $n=6$ & $n=6$ & $n=5$ & $n=5$ & $n=6$ & $n=5$ \\
\hline $\begin{array}{l}\text { Immuno- } \\
\text { fluorescence } \\
\text { staining }\end{array}$ & $n=8$ & $n=8$ & $n=8$ & $n=8$ & $n=7$ & $n=7$ & $n=7$ & $n=7$ \\
\hline
\end{tabular}

Figure 1. Experimental procedure outline. Treadmill exercise preconditioning (EP+) was performed in Sprague-Dawley rats 3 weeks before middle cerebral artery occlusion (MCAO). The filament was removed after $60 \mathrm{~min}$ of MCAO to allow reperfusion. At the onset of reperfusion, separated groups of rats were given intracortical injections of $12.5 \mu \mathrm{g}$ of siRNA-HSP72 or siRNA-vector. Three days after MCAO, neurological function tests, brain water content, Evans blue dye extravasation, and immunohistological examinations were performed. 
(A)

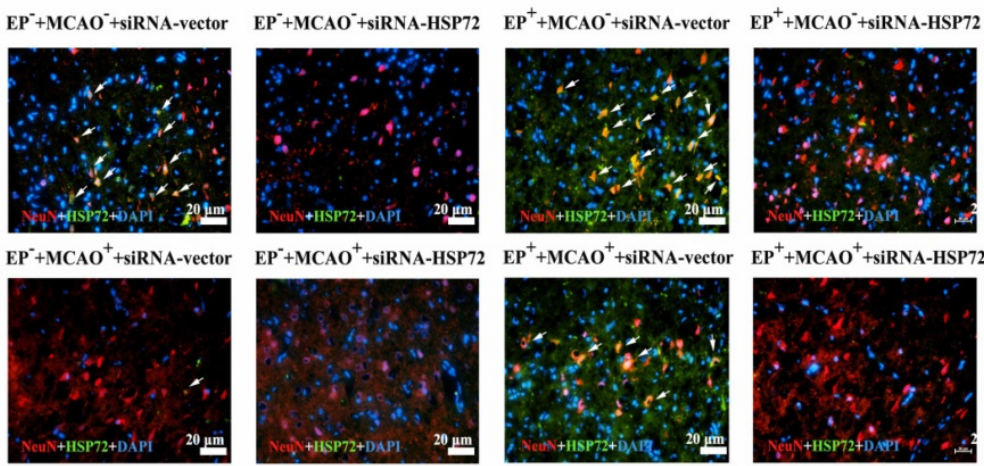

(B)
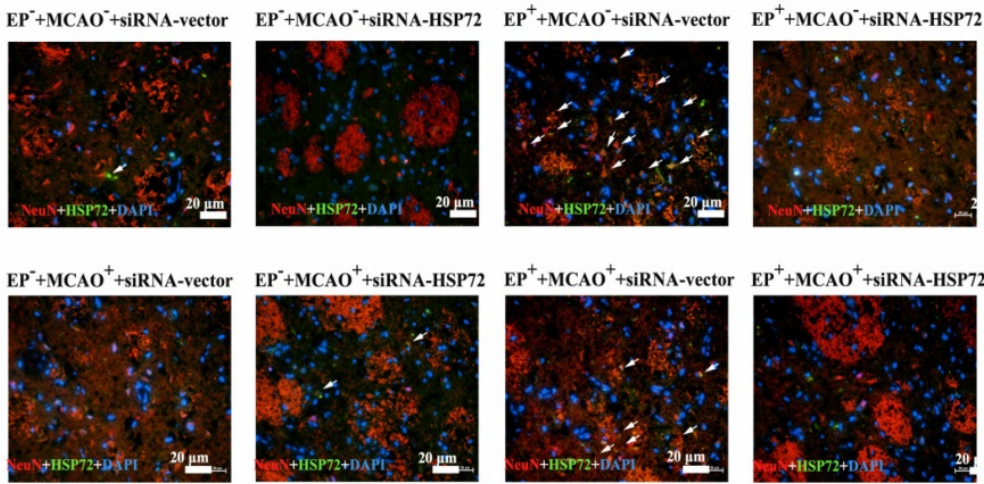

(C)

\section{HSP72-positive neurons}

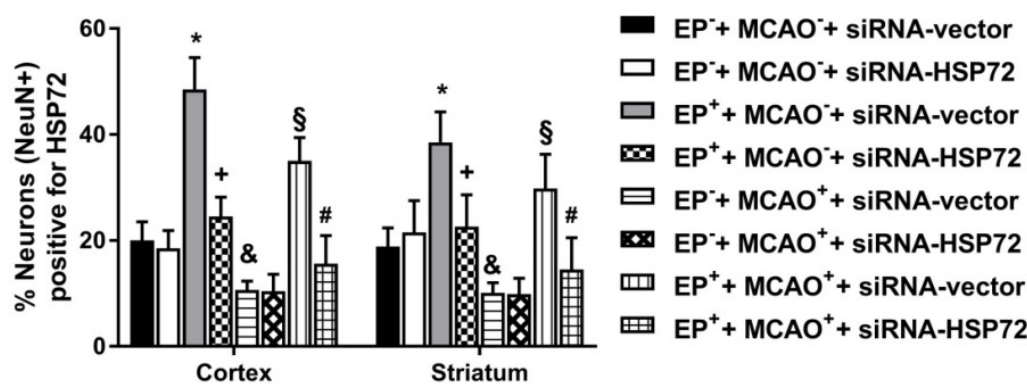

Figure 2. Immunofluorescence detection of heat shock protein (HSP)-72-containing (NeuN+HSP72+DAPI-positive) neurons in the frontal cortex (A) and striatum (B) 3 days after middle cerebral artery (MCAO). For group abbreviations, see the Methods section. Upper panels: the immunofluorescence images are representative of results from different groups of animals. Lower panels: values represent the mean $\pm \mathrm{SD}$ of ten rats per group. $* P<0.05$, EP++MCAO-+siRNA-vector vs. EP-+MCAO-+siRNA-vector; +P<0.05, EP++MCAO-siRNA-HSP72 vs. EP++MCAO-+siRNA-vector; \&P<0.05, EP-+MCAO++siRNA-vector vs. EP-+MCAO-siRNA-vector; $\$ \mathrm{P}<0.05$, EP++MCAO++siRNA-vector vs. EP-+MCAO++siRNA-vector; and \#P<0.05, EP++MCAO++siRNA-HSP72 vs. $\mathrm{EP}^{++} \mathrm{MCAO}^{++}$siRNA-vector. Arrows denote HSP72-containing neurons.

\section{Results}

\section{Cerebral percentages of old HSP72-containing neurons were higher in $\mathrm{EP}+$ rats}

As shown in Figure 2, the percentages of old HSP72-containing neurons (i.e., NeuN+HSP72positive cells) in ipsilateral brain regions, including the frontal cortex and the striatum (Figure $1 a, b$ ), were $\sim 20 \%$ in the EP-+MCAO-+siRNA-vector rats and $10 \%$ in the $\mathrm{EP}^{-}+\mathrm{MCAO}^{+}+$siRNA-vector rats. $\mathrm{EP}^{+}$ significantly increased the percentage of old HSP72-containing neurons to $40-50 \%$ for the $\mathrm{EP}^{+}+\mathrm{MCAO}^{+}+$siRNA-vector rats and $30-35 \%$ for the $\mathrm{EP}^{+}+\mathrm{MCAO}^{-}+\mathrm{siRNA}-$ vector rats (Figure 2). The increased percentage of old HSP72-containing neurons in the ipsilateral brains following $\mathrm{EP}^{+}$was significantly reduced by intracerebral injection of pSUPER siRNA-HSP72 (down to 20-25\%). Compared to the $\mathrm{EP}-\mathrm{MCAO}^{+}+$siRNA-vector rats, the $\mathrm{EP}^{+}+\mathrm{MCAO}^{+}+$siRNA-vector rats had a significantly higher percentage of old HSP72-containing neurons 
$(\sim 35 \%$ vs. $10 \%)$. However, the $\mathrm{EP}^{+}+\mathrm{MCAO}^{++}$ siRNA-HSP72 rats had a significantly lower percentage of old HSP72-containing neurons (5\%) than did the $\mathrm{EP}^{+}+\mathrm{MCAO}^{+}+$siRNA-vector rats.

Figure 3 shows that the percentages of newly formed HSP72-containing neurons (i.e., BrdU+ NeuN+HSP72-positive cells) in ipsilateral brain regions, including the frontal cortex and the striatum, in both the EP-+MCAO-+siRNA-vector and EP-+MCAO-+siRNA-HSP72 rats were within 2-3\%. $\mathrm{EP}^{+}$significantly increased the percentage of newly formed HSP72-containing neurons to $13-15 \%$, as shown in the $\mathrm{EP}^{+}+\mathrm{MCAO}^{-+}+\mathrm{siRNA}-$ vector rats (Figure 3). Additionally, the $\mathrm{EP}^{+}$-induced, newly formed HSP72-containing neurons in the ipsilateral frontal cortex and striatum were significantly reduced by an intracerebral injection of pSUPER siRNA-HSP72 (down to $4-5 \%$ ), as shown in the $\mathrm{EP}^{+}+\mathrm{MCAO}^{-+}$ siRNA-HSP72 rats (Figure 3). Compared to the $\mathrm{EP}-+\mathrm{MCAO}^{+}+$siRNA-vector rats, the $\mathrm{EP}^{+}+\mathrm{MCAO}^{+}+$ siRNA-vector rats had a significantly higher percentage of newly formed HSP72-containing neurons (16-20\% vs. 4-5\%) (Figure 3). Additionally, the increased percentages of newly formed HSP72-containing neurons in both the ipsilateral frontal cortex and striatum were significantly reversed by pSUPER siRNA-HSP72 but not by pSUPER siRNA-vector ( $18 \%$ vs. $\sim 4 \%$ ) (Figure 2$)$ in the $\mathrm{MCAO}^{+}$rats.

(A)
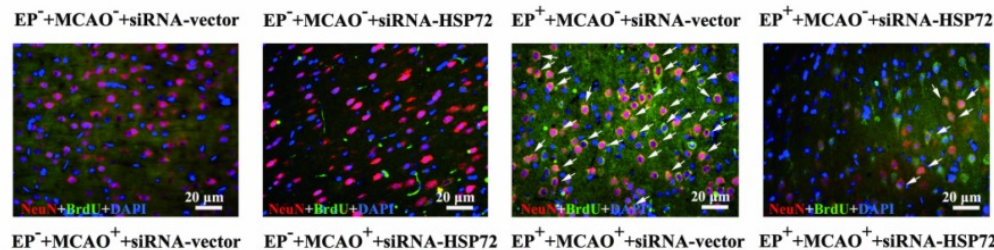

$\mathrm{EP}^{-}+\mathrm{MCAO}^{+}+$siRNA-HS
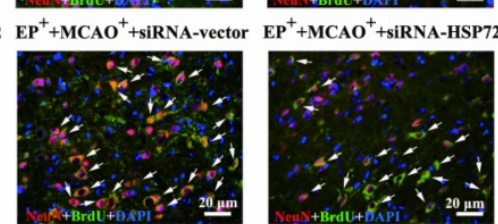

(B)
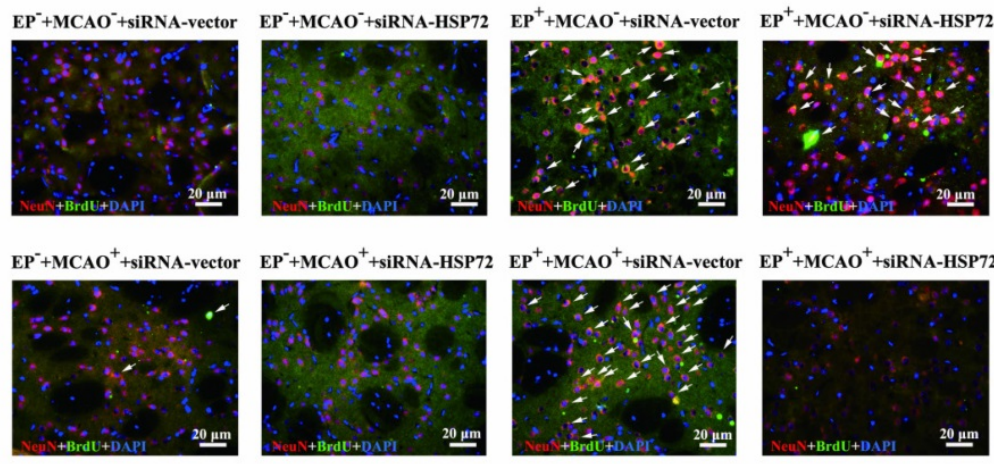

(C)

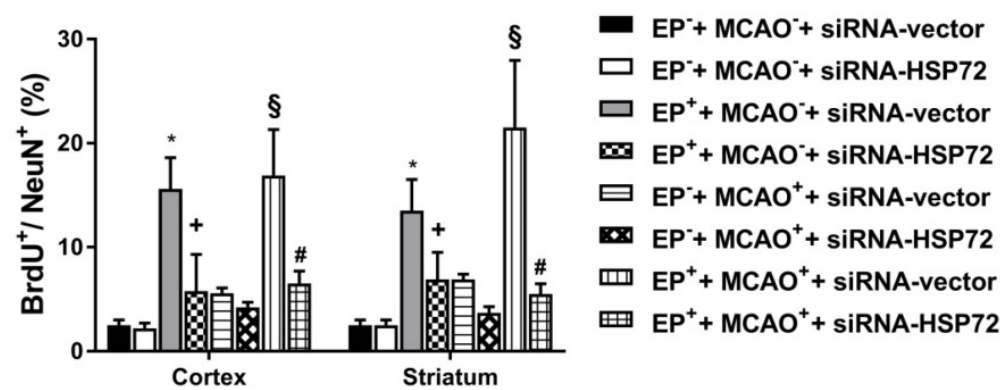

Figure 3. Immunofluorescence detection of newly formed HSP72-containing neurons (BrdU+NeuN+HSP72+DAPI-positive cells) in the frontal cortex (A) and striatum (B) 3 days after MCAO. For group abbreviations, see the Methods section. Upper panels: the immunofluorescence images are representative of results from different groups of animals. Lower panels: values represent the mean $\pm S D$ of ten rats per group. $* P<0.05$, EP++MCAO-+siRNA-vector vs. EP-MCAO-+siRNA-vector; $\quad+\mathrm{P}<0.05, \quad \mathrm{EP}+\mathrm{MCAO}+$ +siRNA-HSP72 vs. EP++MCAO-+siRNA-vector; $\quad \$ P<0.05, \quad E P++M C A O++$ siRNA-vector vs. EP-+MCAO ${ }^{+}+$siRNA-vector; and $\#$ P $<0.05, \mathrm{EP}^{+}+\mathrm{MCAO}^{++}$siRNA-HSP72 vs. EP++MCAO ${ }^{++}$siRNA-vector. Arrows denote newly formed HSP72-containing neurons. 
(A)

\section{mNSS}

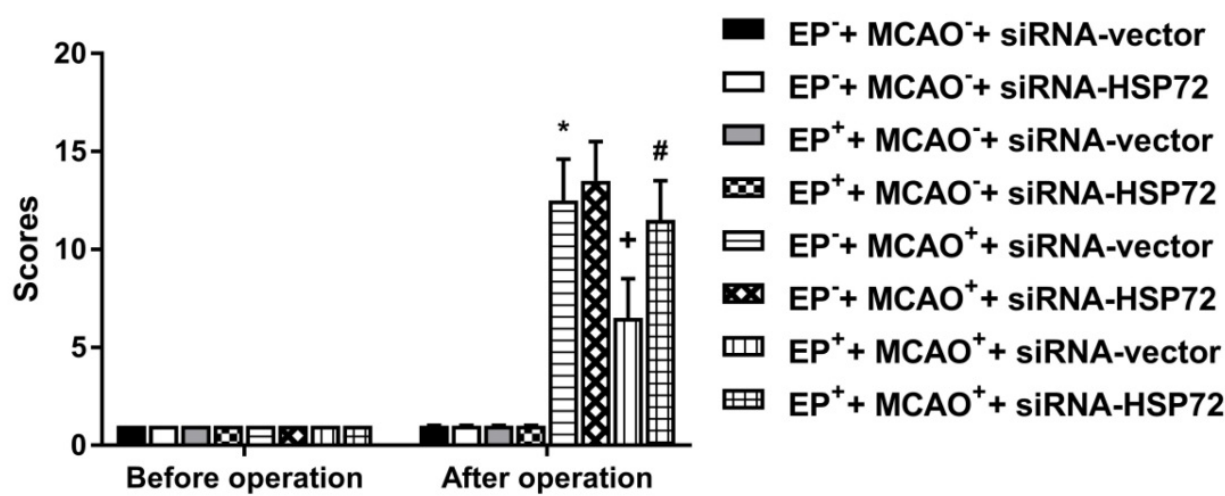

(B)

\section{Inclined plane}
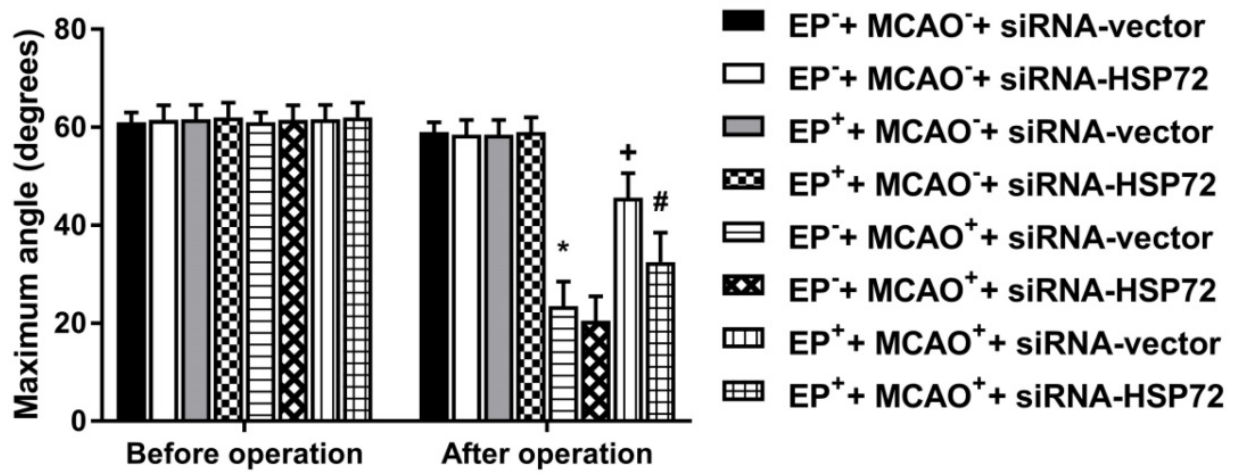

Figure 4. Effects of exercise on cerebral infarct volume in different groups of rats 3 days post-MCAO. Please see the Methods section for group abbreviations. Upper panels: the infarct volume $\left(\mathrm{mm}^{3}\right)$, as revealed by negative 2,3,5-triphenyl tetrazolium chloride staining, indicating dehydrogenase-deficient tissue (pale area), was measured in each slice and summed using computerized planimetry for different groups of rats. Lower panels: values represent the mean \pm SD of eight to ten rats per group.*P<0.01, EP-+MCAO++siRNA-vector vs. EP-+MCAO-+siRNA-vector; $+{ }^{+}<0.05, \mathrm{EP}^{++} \mathrm{MCAO}++$ siRNA-vector vs. EP-+MCAO++siRNA-vector; and \#P<0.05, $\mathrm{EP}^{++} \mathrm{MCAO}^{++}$siRNA-HSP72 vs. $\mathrm{EP}^{++} \mathrm{MCAO}^{++}$siRNA-vector.

\section{Neurological motor deficits after MCAO were lower in EP+ rats}

All EP-+MCAO-+siRNA-vector, EP-+MCAO-+ siRNA-HSP72, $\quad \mathrm{EP}^{+}+\mathrm{MCAO}^{-+}$siRNA-vector and $\mathrm{EP}^{+}+\mathrm{MCAO}^{-}+\mathrm{siRNA}-\mathrm{HSP72}$ rats displayed normal neurological motor function as evidenced by " 0 " mNSS and $60^{\circ}$ maximal angles (Figure 4). Both the $\mathrm{EP}-+\mathrm{MCAO}^{++}$siRNA-vector and $\mathrm{EP}-+\mathrm{MCAO}^{++}$ siRNA-HSP72 groups of rats had significantly $(\mathrm{P}<0.01)$ higher neurological severity scores $(12.5 \pm 2.1$ vs. $2.0 \pm 0.2)$ and lower maximal angles $\left(18 \pm 1^{\circ}\right.$ vs. $\left.60 \pm 3^{\circ}\right)$ than did the EP- control rats. However, the
$\mathrm{EP}^{+}+\mathrm{MCAO}^{+}+$siRNA-vector rats had significantly $(\mathrm{P}<0.05)$ lower neurological severity scores $(6.2 \pm 0.4 \mathrm{vs}$. $12.5 \pm 2.1)$ and higher maximal angles $\left(18 \pm 2^{\circ}\right.$ vs. $\left.6.2 \pm 0.4^{\circ}\right)$ than did the $\mathrm{EP}^{+}$control rats. Additionally, the beneficial effects of $\mathrm{EP}^{+}$in promoting neurological motor recovery following $\mathrm{MCAO}$ were significantly $(\mathrm{P}<0.05)$ reversed by siRNA-HSP72 as shown in the $\mathrm{EP}^{+}+\mathrm{MCAO}^{+}+$siRNA-HSP72 rats $(\mathrm{P}<0.05)$ (Figure 4$)$.

\section{Cerebral infarction and edema after MCAO were attenuated in $\mathrm{EP}^{+}$rats}

All EP-+MCAO-+siRNA-vector, EP-+MCAO-+ siRNA-HSP72, $\quad \mathrm{EP}^{++} \mathrm{MCAO}^{-+}$-siRNA-vector and 
$\mathrm{EP}^{++}+\mathrm{MCAO}^{-+}$-siRNA-HSP72 rats displayed insignificant differences among the groups regarding cerebral infarction (Figure 5), Evans Blue dye extravasation and water content (Figure 6). Both the $\mathrm{EP}-+\mathrm{MCAO}^{+}+$siRNA-vector and $\mathrm{EP}-+\mathrm{MCAO}^{+}+$ siRNA-HSP72 groups had significantly $(\mathrm{P}<0.01)$ greater infarct volume $\left(250 \pm 12 \mathrm{~mm}^{3}\right.$ vs. $\left.0 \mathrm{~mm}^{3}\right)$, increased Evans Blue dye extravasation (1.42 \pm 0.07 $\mu \mathrm{g} / \mathrm{g}$ vs. $0.25 \pm 0.06 \mu \mathrm{g} / \mathrm{g}$ ) and higher water content $(78.5 \pm 1.1 \%$ vs. $73.2 \pm 0.8 \%)$ than did the EP- control rats. However, the $\mathrm{EP}^{+}+\mathrm{MCAO}^{+}+\mathrm{siRNA}-$ vector rats had significantly $(\mathrm{P}<0.05)$ lower brain infarction $(125 \pm 11$ $\left.\mathrm{mm}^{3}\right)$, decreased Evans Blue dye extravasation $(0.68 \pm 0.04 \mu \mathrm{g} / \mathrm{g})$, and lower brain water content $(74.6 \pm 10 \%)$ than did the $\mathrm{EP}^{-}+\mathrm{MCAO}^{+}+$siRNA-vector rats. However, the beneficial effects of $\mathrm{EP}^{+}$in attenuating brain infarction, Evans Blue dye extravasation and brain water content following MCAO were significantly $(\mathrm{P}<0.05)$ reversed by siRNA-HSP72 (Figure 5 and Figure 6) (brain infarct volume, $247 \pm 12 \mathrm{~mm}^{3}$; Evans Blue dye extravasation, $0.68 \pm 0.04 \mathrm{\mu g} / \mathrm{g}$; and brain water content, $78.9 \pm 1.0 \%)$.

(A)

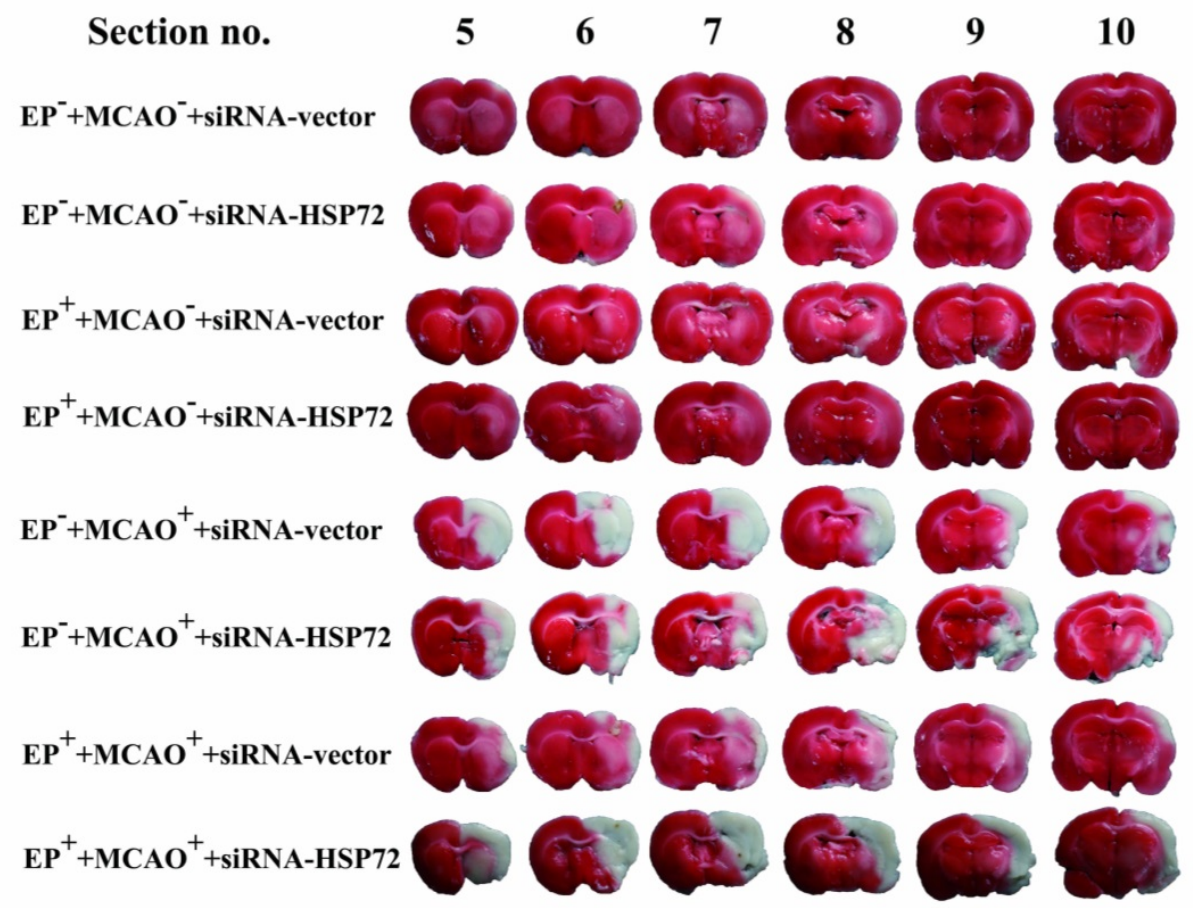

(B)

\section{Infarction volume}
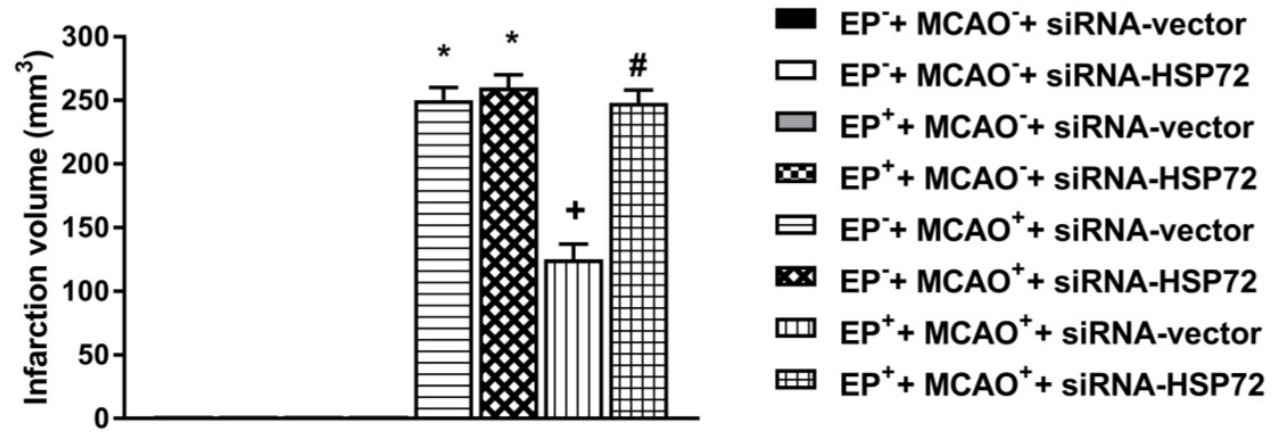

Figure 5. Effects of exercise on both neurological severity scores $(A)$ and maximal angles $(B)$ in different groups of rats 3 days post-MCAO. Data represent the mean $\pm \mathrm{SD}$ (n=8-10 per group). *P<0.01, EP-+MCAO++siRNA-vector vs. EP-+MCAO-+siRNA-vector or EP-+MCAO++siRNA-HSP72 vs. EP-+MCAO-+siRNA-vector;

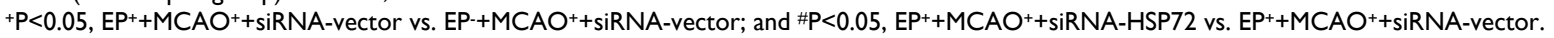




\section{BBB permeability \& brain edema}

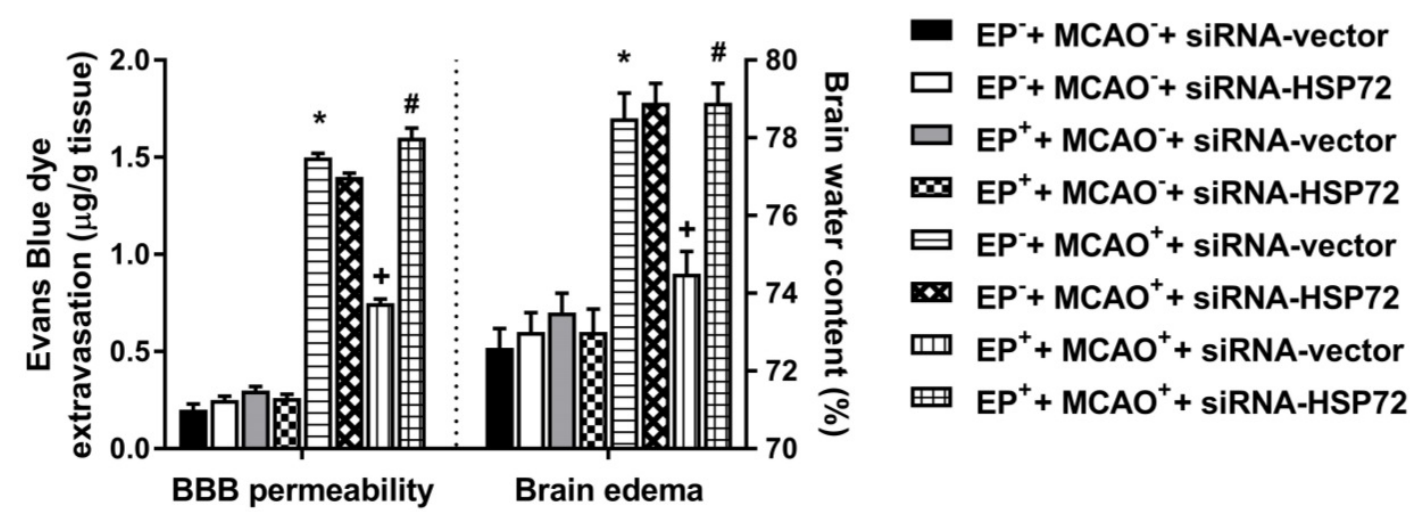

Figure 6. Effects of exercise on both blood-brain barrier (BBB) permeability (i.e., Evans Blue dye extravasation) and brain edema (i.e., brain water content) in different groups of rats 3 days post-MCAO. Data represent the mean \pm SD ( $n=5-6$ per group). *P<0.01, EP-+MCAO++siRNA-vector vs. EP-+MCAO-+siRNA-vector; ${ }^{+} \mathrm{P}<0.05, \mathrm{EP}^{+}+\mathrm{MCAO}^{++}$siRNA-vector vs. EP-+MCAO++siRNA-vector; $\#{ }^{+}<0.05, \mathrm{EP}^{++} \mathrm{MCAO}^{++}$siRNA-HSP72 vs. EP++MCAO++siRNA-vector.

\section{Discussion}

The most striking findings of the present study are that $\mathrm{EP}^{+}$provides significant neuroprotection against ischemic brain injury by preserving both old HSP72-containing neurons and newly formed HSP72-containing neurons. Our present results are consistent with many previous findings. For example, both in vitro cerebral ischemic injury and in vivo spinal cord ischemic injury can be ameliorated by transfection and viral overexpression of HSP72 in neurons and glia. Mice and rats overexpressing HSP72 are protected against cerebral infarction [25-27]. Knockout of HSP72 worsens ischemic brain injury in rodents with focal brain ischemia [28]. Geldanamycin induces HSP72 in the brain and protects against focal ischemic brain injury in rats [29, 30].

Previous studies have demonstrated that $\mathrm{EP}^{+}$ induced a $70 \%$ higher expression of HSP72 in the ischemic brain and diminished neuronal injury in rats with MCAO [6]. Antibody inhibition of $\mathrm{EP}^{+}$-induced mild HSP72 expression in the ischemic brain completely blocked $\mathrm{EP}^{+}$-induced neuroprotection. This result indicates that the increased HSP72 levels prior to ischemia and reperfusion brain injury may be one of the key issues. In the present study, in sham-operated rats (i.e., $\mathrm{MCAO}^{-}$rats), $\mathrm{EP}^{+}$increased the percentage of both old HSP72-containing neurons (from $19 \%$ to $45 \%$ ) and newly formed HSP72-containing neurons (from 2.5\% to 16\%) (Figure $3 a, 3 b)$. Intracerebral injection of pSUPER siRNA-HSP72 significantly reduced the percentage of both old and newly formed HSP72-containing neurons to $25 \%$ and $10 \%$, respectively. At the same time, $\mathrm{MCAO}^{+}$significantly attenuated the percentage of old and newly formed HSP72-containing neurons to $10 \%$ and $5 \%$, respectively. The reduction of HSP72-containing neurons in MCAO rats was blocked by $\mathrm{EP}^{+}(30 \%$ and $18 \%$, respectively, for old and newly formed HSP72-containing neurons). The beneficial effects of $\mathrm{EP}^{+}$in preserving the old HSP72-containing neurons and increasing the newly formed HSP72-containing neurons in ipsilateral brain tissues were attenuated by intracerebral injection of siRNA-HSP72 to $15 \%$ and $4.5 \%$, respectively. These results suggest that HSP72 antibody or siRNA-HSP72 gene silencing had an effect by neutralizing HSP72 or reducing the accumulation of HSP72-containing neurons produced by $\mathrm{EP}^{+}$in the brain, leading to interference of an anti-neural injury mechanism.

$\mathrm{EP}^{+}$provides significant protection against acute cerebral ischemic injury (present results and [31]). $\mathrm{EP}^{+}$ induces brain ischemic tolerance through the promotion of angiogenesis, mediation of inflammatory responses, and inhibition of neuronal apoptosis. $\mathrm{EP}^{+}$-mediated overexpression of cerebral HSP72 improves outcomes of severe ischemic injury via increasing brain derived neurotrophic factor (BDNF) production and/or decreasing neuroinflammation and neuronal apoptosis (via elevated Bcl-2/Bax ratio, preventing formation of the apoptosome, and inhibition of caspase-3) [12, 32-34]. Cerebral overexpression of HSP72 exerts both anti-inflammatory and antiapoptotic effects and facilitates neuroprotection. In mice with comparable cerebral ischemic injury, overexpression of HSP72 reduced the density of glial fibrillary acidic protein (GFAP)-positive cells and decreased astrocyte morphological complexity [35]. In addition, HSP72-mediated $\mathrm{EP}^{+}$may attenuate BBB disruption and cerebral ischemic injury in rats via inhibiting the 
matrix metalloproteinase-9 (MMP-9) [36]/nuclear factor NF- $\mathrm{B}$ [6] pathway. Furthermore, HSP72-mediated preconditioning exercise might reduce cerebral ischemic injury and neuronal apoptosis through endogenous 14-3-3 $\gamma$ [31].

\section{Conclusion}

In conclusion, a significant $(\mathrm{P}<0.05)$ increase in the percentages of both old and newly formed HSP72-containing neurons after 3 weeks of exercise coincided with a significant $(\mathrm{P}<0.05)$ reduction in brain infarct volume, brain edema, BBB disruption, and neurological motor deficits in cerebral ischemic injury rats. Reductions in the percentages of both the old and newly formed HSP72-containing neurons by pSUPER siRNA showed a significant $(\mathrm{P}<0.05)$ reversal in the neuroprotective effects of $\mathrm{EP}^{+}$. Our data provide an inverse correlation between the $\mathrm{EP}^{+}$-mediated upregulation of old and newly formed HSP72-containing neurons and the extent of cerebral ischemic injury. Our data demonstrate that preischemic treadmill exercise improves cerebral ischemic injury by preserving the percentages of both old HSP72-containing neurons and newly formed HSP72-containing neurons.

\section{Abbreviations}

$\mathrm{EP}^{+}$: exercise preconditioning; HSP72: heat shock protein 72; siRNA: small interfering RNA; BrdU: bromodeoxyuridine; NeuN: neuronal nuclei; pSUPER: suppression of endogenous RNA; MCAO: middle cerebral artery occlusion; TTC: 2,3,5-triphenyltetrazolium chloride; PBS: phosphate-buffered saline; DAPI: 4',6-diamidino-2-phenylindole.

\section{Acknowledgments}

This study is partially supported by the Ministry of Science and Technology (Taiwan) under grant no. MOST 107-2314-B-384-007-MY3, grant no. MOST 108-2314-B-715-001, and the Chi Mei Medical Center, Tainan, Taiwan under grant no. CMFHT10401.

\section{Author contributions}

Study design: Fong-Chin Su, Willy Chou

Performed the experiments \& data collection: Yu-Ling Wang, Cheng-Hsien Lin, Chi-Chun Chen

Statistical analysis: Ching-Ping Chang,

Kao-Chang Lin

Data interpretation: Yu-Ling Wang, Willy Chou Chou

Manuscript preparation: Fong-Chin Su, Willy

Literature search: Yu-Ling Wang, Ching-Ping Chang, Kao-Chang Lin

\section{Competing Interests}

The authors have declared that no competing interest exists.

\section{References}

1. Goldstein LB, Bushnell CD, Adams RJ, et al. Guidelines for the primary prevention of stroke: a guideline for healthcare professionals from the American Heart Association/American Stroke Association. Stroke. 2011; 42: 517-84.

2. Wolf PA, Clagett GP, Easton JD, et al. Preventing ischemic stroke in patients with prior stroke and transient ischemic attack : a statement for healthcare professionals from the Stroke Council of the American Heart Association. Stroke. 1999; 30: 1991-4.

3. Reinholdsson M, Palstam A, Sunnerhagen KS. Prestroke physical activity could influence acute stroke severity (part of PAPSIGOT). Neurology. 2018; 91: e1461-e67.

4. Rist PM, Capistrant BD, Mayeda ER, et al. Physical activity, but not body mass index, predicts less disability before and after stroke. Neurology. 2017; 88: 1718-26.

5. Zhao JH, Meng XL, Zhang J, et al. Oxygen glucose deprivation post-conditioning protects cortical neurons against oxygen-glucose deprivation injury: role of HSP70 and inhibition of apoptosis. J Huazhong Univ Sci Technolog Med Sci. 2014; 34: 18-22.

6. Sharp FR, Zhan X, Liu D-Z. Heat shock proteins in the brain: role of Hsp70, Hsp 27, and HO-1 (Hsp32) and their therapeutic potential. Translational stroke research. 2013; 4: 685-92.

7. Sharp FR, Lu A, Tang Y, et al. Multiple molecular penumbras after focal cerebral ischemia. J Cereb Blood Flow Metab. 2000; 20: 1011-32.

8. Chang $\mathrm{CK}$, Chou $\mathrm{W}$, Lin $\mathrm{HJ}$, et al. Exercise preconditioning protects against spinal cord injury in rats by upregulating neuronal and astroglial heat shock protein 72. Int J Mol Sci. 2014; 15: 19018-36.

9. Zhan X, Ander BP, Liao IH, et al. Recombinant Fv-Hsp70 protein mediates neuroprotection after focal cerebral ischemia in rats. Stroke. 2010; 41: 538-43.

10. Giffard RG, Han R-Q, Emery JF, et al. Regulation of apoptotic and inflammatory cell signaling in cerebral ischemia: the complex roles of heat shock protein 70. Anesthesiology. 2008; 109: 339-48.

11. Mohanan A, Deshpande S, Jamadarkhana PG, et al. Delayed intervention in experimental stroke with TRC051384--a small molecule HSP70 inducer. Neuropharmacology. 2011; 60: 991-9.

12. Chio CC, Lin HJ, Tian YF, et al. Exercise attenuates neurological deficits by stimulating a critical HSP70/NF-kappaB/IL-6/synapsin I axis in traumatic brain injury rats. J Neuroinflammation. 2017; 14: 90 .

13. Lin $\mathrm{CM}$, Chang $\mathrm{CK}$, Chang $\mathrm{CP}$, et al. Protecting against ischaemic stroke in rats by heat shock protein 20-mediated exercise. Eur J Clin Invest. 2015; 45: 1297-305.

14. Brummelkamp TR, Bernards R, Agami R. A system for stable expression of short interfering RNAs in mammalian cells. Science. 2002; 296: 550-3.

15. Hoehn BD, Palmer TD, Steinberg GK. Neurogenesis in rats after focal cerebral ischemia is enhanced by indomethacin. Stroke. 2005; 36: 2718-24

16. Paxinos G, Watson C. The rat brain in stereotaxic coordinates. 7th ed. New York Elsevier Academic Press. 2013

17. Chen $\mathrm{SF}$, Hsu $\mathrm{CW}$, Huang $\mathrm{WH}$, et al. Post-injury baicalein improves histological and functional outcomes and reduces inflammatory cytokines after experimental traumatic brain injury. Br J Pharmacol. 2008; 155: 1279-96.

18. Chang MW, Young MS, Lin MT. An inclined plane system with microcontroller to determine limb motor function of laboratory animals. J Neurosci Methods. 2008; 168: 186-94.

19. Bederson JB, Pitts LH, Germano SM, et al. Evaluation of 2,3,5-triphenyltetrazolium chloride as a stain for detection and quantification of experimental cerebral infarction in rats. Stroke. 1986; 17: 1304-8.

20. Gartshore G, Patterson J, Macrae IM. Influence of ischemia and reperfusion on the course of brain tissue swelling and blood-brain barrier permeability in a rodent model of transient focal cerebral ischemia. Exp Neurol. 1997; 147: $353-60$.

21. Lin TN, He YY, Wu G, et al. Effect of brain edema on infarct volume in a focal cerebral ischemia model in rats. Stroke. 1993; 24: 117-21.

22. Zhang RL, Zhang ZG, Zhang L, et al. Proliferation and differentiation of progenitor cells in the cortex and the subventricular zone in the adult rat after focal cerebral ischemia. Neuroscience. 2001; 105: 33-41.

23. Huang $B$, Krafft $P R, M a ~ Q$ et al. Fibroblast growth factors preserve blood-brain barrier integrity through RhoA inhibition after intracerebral hemorrhage in mice. Neurobiol Dis. 2012; 46: 204-14.

24. Lin HJ, Hsu CC, Chio CC, et al. Gamma-Secretase Inhibitors Attenuate Neurotrauma and Neurogenic Acute Lung Injury in Rats by Rescuing the Accumulation of Hypertrophic Microglia. Cell Physiol Biochem. 2017; 44: 1726-40.

25. van der Weerd L, Lythgoe MF, Badin RA, et al. Neuroprotective effects of HSP70 overexpression after cerebral ischaemia--an MRI study. Exp Neurol. 2005; 195: 257-66.

26. Rajdev S, Hara K, Kokubo Y, et al. Mice overexpressing rat heat shock protein 70 are protected against cerebral infarction. Ann Neurol. 2000; 47: 782-91. 
27. Hoehn B, Ringer TM, Xu L, et al. Overexpression of HSP72 after induction of experimental stroke protects neurons from ischemic damage. J Cereb Blood Flow Metab. 2001; 21: 1303-9.

28. Lee SH, Kwon HM, Kim YJ, et al. Effects of hsp70.1 gene knockout on the mitochondrial apoptotic pathway after focal cerebral ischemia. Stroke. 2004; 35: 2195-9.

29. Kwon HM, Kim Y, Yang SI, et al. Geldanamycin protects rat brain through overexpression of HSP70 and reducing brain edema after cerebral focal ischemia. Neurol Res. 2008; 30: 740-5.

30. Lu A, Ran R, Parmentier-Batteur S, et al. Geldanamycin induces heat shock proteins in brain and protects against focal cerebral ischemia. J Neurochem. 2002; 81: 355-64.

31. Otsuka S, Sakakima H, Terashi T, et al. Preconditioning exercise reduces brain damage and neuronal apoptosis through enhanced endogenous 14-3-3gamma after focal brain ischemia in rats. Brain Struct Funct. 2018; https://doi.org/10.1007/s00429-018-1800-4

32. Otsuka S, Sakakima $H$, Sumizono $M$, et al. The neuroprotective effects of preconditioning exercise on brain damage and neurotrophic factors after focal brain ischemia in rats. Behav Brain Res. 2016; 303: 9-18.

33. Hayes K, Sprague S, Guo M, et al. Forced, not voluntary, exercise effectively induces neuroprotection in stroke. Acta Neuropathol. 2008; 115: 289-96.

34. Koo JH, Kwon IS, Kang EB, et al. Neuroprotective effects of treadmill exercise on BDNF and PI3-K/Akt signaling pathway in the cortex of transgenic mice model of Alzheimer's disease. Journal of exercise nutrition \& biochemistry. 2013; 17: 151-60.

35. Barreto GE, White RE, Xu L, et al. Effects of heat shock protein 72 (Hsp72) on evolution of astrocyte activation following stroke in the mouse. Exp Neurol. 2012; 238: 284-96.

36. Chaudhry K, Rogers R, Guo M, et al. Matrix metalloproteinase-9 (MMP-9) expression and extracellular signal-regulated kinase 1 and 2 (ERK1/2) activation in exercise-reduced neuronal apoptosis after stroke. Neurosci Lett. 2010; 474: 109-14. 